\title{
Phenethyl isothiocyanate suppresses cervical carcinoma metastasis potential and its molecular mechanism
}

\author{
LI ZHANG, QUAN HAO, LEWEN BAO, WENXIN LIU, XIN FU, YING CHEN and HUIJUAN WU \\ Department of Gynecologic Cancer, Tianjin Medical University Cancer Institute and Hospital, \\ National Clinical Research Center for Cancer, Key Laboratory of Cancer Prevention and Therapy, \\ Tianjin 300060, P.R. China
}

Received December 2, 2013; Accepted July 31, 2014

DOI: $10.3892 / \mathrm{mmr} .2014 .2565$

\begin{abstract}
Tumor metastasis is a prominent cause of treatment failure in cervical carcinoma. Phenethyl isothiocyanate (PEITC) is an active component extracted from cruciferous plants that has exhibited anticancer activity in various types of human cancer; however, its effect on the inhibition of metastasis remains unclear. The current study aimed to explore the effect of PEITC on the suppression of metastasis in HeLa cervical carcinoma cells. Multiple variables were assessed with different methods as follows: Cell viability, with a Vi-CELL analyzer; cell adhesion, by MTS assay; cell invasion, by Transwell assay; cell cycle, by flow cytometry assay; cytokine concentration, by ELISA assay; metastasis-related gene and protein expression, by quantitative polymerase chain reaction and western blotting; and transcription factor activity, by gene reporter assay. The results indicated that PEITC exhibited an inhibitory effect on the adhesion and invasion of HeLa cells by induction of $\mathrm{G}_{2} / \mathrm{M}$ phase arrest, it reduced the expression of CDK1, MMP-2/9, CD44, ICAM-1, increased the production of TGF- $\beta$, IL- 6 and IL- 8 , and increased the phosphorylation of Smad2. These results suggest that PEITC may be a potential antitumor compound, acting through the TGF- $\beta / \mathrm{Smad} 2$ pathway; and it has the potential for future use as a therapy for cervical carcinoma subsequent to further studies.
\end{abstract}

\section{Introduction}

Cervical cancer is the third most commonly diagnosed cancer and the fourth leading cause of cancer-associated mortality in females worldwide, accounting for $9 \%$ of the total cancer

Correspondence to: Mr. Quan Hao, Department of Gynecologic Cancer, Tianjin Medical University Cancer Institute and Hospital, National Clinical Research Center for Cancer, Key Laboratory of Cancer Prevention and Therapy, No. 1 Huanhuxi Road, Hexi District, Tianjin 300060, P.R. China

E-mail: haoquan_muc@163.com

Key words: phenethyl isothiocyanate, cervical carcinoma, tumor metastasis diagnoses and $8 \%$ of the total cancer-related mortalities among females in 2008 ; $>85 \%$ of these cases occur in developing countries (1). The frequency of lymph node metastasis increases with clinical stage; and invasive cervical cancer is a serious problem and a leading cause of cancer-related mortality in women (2). The majority of patients with cervical cancer receive standard radiotherapy and chemotherapy, but clinical outcomes vary significantly and are difficult to predict.

It has been established that invasion and adhesion are fundamental properties of malignant cancer cells (3). The formation of metastatic nodules involves multiple mechanisms, including the loss of cellular adhesion; increased motility and invasiveness; entry to and survival in the circulatory system, exit to new tissue; and the eventual colonization of a distant metasasis site. A wide variety of factors contribute to the metastasis of tumor cells, including cytokines, hormones, growth factors, cell adhesion molecules and matrix metalloproteinases (MMPs). Therefore, natural agents that are able to suppress the expression of these factors may be considered for development as treatments to prevent cervical cancer invasion and metastasis (4).

Isothiocyanates are natural compounds located in consumable cruciferous vegetables such as broccoli, watercress, brussel sprouts, cabbage, Japanese radishes and cauliflower. They have been indicated to inhibit the chemical carcinogenesis caused by a wide variety of chemical carcinogens in animal models (5). Previous studies have also demonstrated that isothiocyanates exhibit antitumor activity, inhibiting growth and metastasis in various human cancer cell models. Other studies have revealed that phenethyl isothiocyanate (PEITC) suppresses the growth of cancer cells in vitro and in vivo (6). Several cellular responses to this potential chemopreventative agent have been previously described, including apoptosis mediated by c-Jun amino terminal kinase and extracellular signal-regulated kinase $(7,8)$. However, the mechanisms behind the anticancer effects of PEITC are not clearly understood.

Although numerous studies have indicated that PEITC can be used as an inducer of apoptosis, there are thus far no reports implicating PEITC in the inhibition of the metastasis potential of cervical cancer cells. Therefore, the present study analyzed the inhibitory effects of PEITC on the metastatic potential of cervical cancer cells. HeLa cervical carcinoma cells were used to examine the effect of PEITC on cell proliferation, invasion, migration and the expression of metastasis-related genes. 


\section{Materials and methods}

Cell culture. The human HeLa cervical carcinoma cell line was obtained from the American Type Culture Collection (Manassas, VA, USA). The cells were grown and maintained in RPMI-1640 medium supplemented with $10 \%$ fetal bovine serum (FBS) and $2 \mathrm{mM}$ glutamine (Gibco Life Technologies, Carlsbad, CA, USA) at $37^{\circ} \mathrm{C}, 5 \% \mathrm{CO}_{2}$. Penicillin and streptomycin were not added to the culture medium to avoid interaction with isothiocyanates. A stock solution of $1 \mathrm{mM}$ PEITC dissolved in DMSO (Sigma-Aldrich, St. Louis, MO, USA) was made and added to cell medium at various concentrations for the treatment of cells.

Cell proliferation assay. Cells were seeded at a density of $2 \times 10^{3}$ into a 96-well plate overnight and incubated with $0,0.1,0.2$, $0.5,1,2,5,10,20,50$ and $100 \mu \mathrm{M}$ PEITC for $24 \mathrm{~h}$ at $37^{\circ} \mathrm{C}$. The cell viability was determined with a Vi-CELL cell viability analyzer (Beckman Coulter, Brea, CA, USA) according to the manufacturer's instructions, and the $\mathrm{IC}_{50}$ values were calculated.

Adhesion assay. Matrigel was purchased from BD Biosciences (Franklin Lakes, NJ, USA) and a concentration was used according to the manufacturer's instructions. The confluent cultures of $2 \times 10^{5}$ cells were plated onto the Matrigel-coated insert of the 96-well culture plates and incubated with 5 or $10 \mu \mathrm{M}$ PEITC for $6 \mathrm{~h}$ in standard culture medium. Next, the liquid was discarded and the cells were gently washed once with PBS. The cells were then analyzed by MTS assay (Promega Corporation, Madison, WI, USA). Briefly, $20 \mu \mathrm{l}$ MTS was added to the fresh culture medium and incubated for $4 \mathrm{~h}$, then the absorbance was determined using a Synergy 2 Multi-Mode Microplate Reader (BioTek Instruments, Inc., Shoreline, WA, USA) at $490 \mathrm{~nm}$ (A490) to calculate the percentage cell viability. The background absorbance was measured at $630 \mathrm{~nm}$ (A630).

Cell invasion assay. A cell invasion assay was performed using Transwell cell culture inserts (Invitrogen Life Technologies, Carlsbad, CA, USA). Briefly, $3 \times 10^{4}$ cells were harvested, resuspended in serum-free medium, and then transferred to the hydrated Matrigel (BD Biosciences) upper chambers. The chambers were then incubated with 5 and $10 \mu \mathrm{M}$ PEITC for $24 \mathrm{~h}$ in culture medium with $10 \% \mathrm{FBS}$, then the invaded cells on the lower surface were fixed and stained with $0.05 \%$ crystal violet (Sigma-Aldrich) for $2 \mathrm{~h}$. Finally, the invaded cells were counted under a microscope at a magnification of $x 40$ and the relative number of invaded cells to total cells was calculated.

Cell cycle analysis. Cells were cultured in 6-well plates at a density of $3 \times 10^{5}$, in medium without FBS for $24 \mathrm{~h}$ for synchronization, then incubated with $5 \mu \mathrm{M}$ and $10 \mu \mathrm{M}$ PEITC for a further $24 \mathrm{~h}$. The cells were harvested using trypsin (Sigma-Aldrich) and fixed in $70 \%$ cold ethanol overnight at $4^{\circ} \mathrm{C}$. The cells were then resuspended in $1 \mathrm{ml}$ PBS containing $1 \mathrm{mg} / \mathrm{ml}$ RNase A Solution and $0.5 \mathrm{mg} / \mathrm{ml}$ Propidium Iodide (Sigma-Aldrich) for $30 \mathrm{~min}$. Next, $1 \times 10^{4}$ events were collected and analyzed by flow cytometry (BD Biosciences). Modfit LT software, version 3.0 (Verity Software House, Inc., Topsham, ME, USA) was used to analyze the results.
ELISA analysis. The ELISA kits (R\&D Systems, Inc., Minneapolis, MN, USA) were used to detect the concentration of TGF- $\beta$, IL- 6 and IL- 8 in the cell culture supernatant, according to the manufacturer's instructions. Briefly, $3 \times 10^{5}$ cells were cultured in 6-well plates overnight and incubated with 5 and $10 \mu \mathrm{M}$ PEITC for $24 \mathrm{~h}$, then the culture was replaced with DMEM without FBS for $24 \mathrm{~h}$, and the cell culture supernatant was collected. A total of $100 \mu \mathrm{l}$ of the supernatant was added to microplate wells, and $200 \mu 1$ anti-TGF- $\beta$, anti-IL-6 and anti-IL- 8 were added $10 \mathrm{sec}$ later. Subsequent to incubation at $37^{\circ} \mathrm{C}$ for $30 \mathrm{~min}$, the sample was washed 5 times with wash buffer, followed by the addition of $200 \mu 1$ horseradish peroxidase (Santa Cruz Biotechnology, Dallas, TX, USA) to each well following incubation at $37^{\circ} \mathrm{C}$ for $30 \mathrm{~min}$. Next, the wells were washed 5 times with Tris-buffered saline with Tween $20(20 \mathrm{~nm}$ Tris, pH 7.5, $150 \mathrm{nM} \mathrm{NaCl}, 0.1 \%$ Tween 20), followed by the addition of $100 \mu 1$ tetramethylbenzidine (Sigma-Aldrich) and incubated at room temperature in the dark. After a 20 min incubation, $100 \mu \mathrm{l}$ stop solution was added, and the absorbance was read on a microplate reader at $450 \mathrm{~nm}$. All experiments were performed in duplicate. The values were calculated from standard curves.

Quantitative polymerase chain reaction $(q P C R)$. A total of $3 \times 10^{5}$ cells were cultured in a 6 -well plate overnight and incubated with 5 or $10 \mu \mathrm{M}$ PEITC for $24 \mathrm{~h}$, then total cellular RNA was isolated with TRIzol ${ }^{\circledast}$ reagent (Invitrogen Life Technologies) according to the manufacturer's instructions. cDNA for each cell line was synthesized from $1 \mu \mathrm{g}$ total RNA and SuperScript Reverse Transcriptase as described in the manufacturer's protocol (Takara Bio, Inc., Otsu, Japan). qPCR assays were performed by SYBR-Green (Takara Bio, Inc.) incorporation. The relative gene expression was determined by cycle threshold calculation, utilizing actin for normalization and GAPDH as a reference gene. The following primers were used to amplify the cDNA fragments: CD44 F 5'-CAGATGGCC CATACCTTCAAAT-3' and R 5'-CGGAAACGAAATCCT CTCTGTT-3'; ICAM-1, F 5'-CTCCAATGTGCCAGG CTTG-3' and R 5'-CAGTGGGAAAGTGCCATCCT-3'; MMP-2 F 5'-CTTCCAAGTCTGGAGCGATGT-3' and R 5'-TACCGTCAAAGGGGTATCCAT-3'; MMP-9 F 5'-GGGACGCAGACATCGTCATC-3' and R 5'-TCG TCATCGTCGAAATGGGC-3'; CDK1 F 5'-TTCAGG ATGTGCTTATGC-3' and R 5'-AGAGCAATTCCA AGCCAT-3'; GAPDH F 5'-TGTTGCCATCAATGACCC CTT-3' and R 5'-CTCCACGACGTACTCAGCG-3. The PCR cycling conditions were as follows: Denaturation at $95^{\circ} \mathrm{C}$ for $60 \mathrm{sec}, 40$ cycles of $95^{\circ} \mathrm{C}$ for $5 \mathrm{sec}$ and hybridization at $72^{\circ} \mathrm{C}$ for $60 \mathrm{sec}$. GAPDH was used as an internal control for loading.

Western blotting. A total of $3 \times 10^{5}$ cells were cultured in 6-well plates overnight and incubated with 5 or $10 \mu \mathrm{M}$ PEITC for $24 \mathrm{~h}$, then cells were lysed with $1 \mathrm{X}$ modified radioimmunoprecipitation assay buffer $(50 \mathrm{mM}$ Tris, $150 \mathrm{mM} \mathrm{NaCl}, 1 \%$ Triton $\mathrm{X}-100$, and $0.5 \%$ deoxycholate) containing $25 \mu \mathrm{g} / \mathrm{ml}$ leupeptin (Sigma-Aldrich), $10 \mu \mathrm{g} / \mathrm{ml}$ aprotinin (Sigma-Aldrich), $1 \mathrm{mM}$ sodium orthovanadate and $2 \mathrm{mM}$ EDTA. The protein concentrations of the samples were determined using a Bicinchoninic Acid Protein Assay Reagent kit (Qiagen, Valencia, CA, USA), and the cell lysates were analyzed by $8 \%$ SDS-PAGE. The 
samples were then transferred to polyvinylidene fluoride membranes (EMD Millipore, Billerica, MA, USA). The blots were blocked for $1 \mathrm{~h}$ at room temperature with $5 \%$ milk and incubated with the following monoclonal antibodies: CD44, (1:800); ICAM-1 (1:800); MMP-2 (1:800); MMP-9 (1:500); CDK1 (1:500); and p-Smad2 (1:500) (Santa Cruz Biotechnology) and mouse $\beta$-actin (1:2,000; Sigma-Aldrich) which was used for protein loading analyses. The blots were developed using an enhanced chemiluminescence (ECL) Plus Western Blotting Detection Reagents kit (GE Healthcare Bio-Sciences, Pittsburgh, PA, USA). The intensity of the protein bands was determined by densitometry using a Gene Genius Super system (Gene Tech Co., Ltd., Hong Kong, China).

Statistical analysis. The data are presented as the means \pm standard deviation. All the data from the current study were evaluated using SPSS software, version 11.5 (SPSS, Inc., Chicago, IL, USA). Assessment of the differences between groups was performed by one-way analysis of variance. $\mathrm{P}<0.05$ was considered to indicate a statistically significant difference.

\section{Results}

Effect of PEITC on the proliferative potential of HeLa cells. To study the effect of PEITC on the growth of cervical carcinoma cells, HeLa cells were incubated with various concentrations of PEITC. The results indicated a dose-dependent inhibition of cell growth; the $\mathrm{IC}_{50}$ value was $31.3 \mu \mathrm{M}$ and the proliferation inhibition rates of 24 -h treatment with 5 and $10 \mu \mathrm{M}$ PEITC were 8.4 and $12.6 \%$. These concentrations were selected as no-toxity $(<10 \%)$ and low-toxity $(<15 \%)$ doses for the adhesion, invasion, cell cycle, cytokine, western blotting, qPCR and reporter gene assays, to avoid interference of toxity with the results (Fig. 1).

PEITC reduces the adhesive potential of HeLa cells. The effect of PEITC on the adhesion of HeLa cells was assessed by adhesion assay. When HeLa cells were incubated with PEITC, cellular adhesion was inhibited, and the adhesion rates were reduced to 71.2 and $55.7 \%$ of the control group following 24-h treatment with 5 and $10 \mu \mathrm{M}$ PEITC $(\mathrm{P}<0.05)$, respectively (Fig. 2).

PEITC reduces the invasive potential of HeLa cells. Invasion is another important step for metastasis, so the inhibitory effect of PEITC on the ability of HeLa cells to invade a reconstituted extracellular matrix was investigated. It was demonstrated that PEITC inhibited cell invasion in a dose-dependent manner. When HeLa cells were grown on Matrigel, a significant reduction in the number of invading cells was observed following treatment with PEITC. The levels of invasion were reduced to 65.2 and $21.7 \%$ of control levels by 5 and $10 \mu \mathrm{M}$ PEITC treatment $(\mathrm{P}<0.05)$, respectively (Fig. 3$)$.

PEITC treatment leads to $G_{2} / M$ phase cell cycle arrest in HeLa cells. Cell cycle phase assay was performed by flow cytometry, subsequent to staining of the fixed cells with PI. As displayed in Fig. 4, PEITC markedly induced $\mathrm{G}_{2} / \mathrm{M}$ arrest in HeLa cells; the percentages of $\mathrm{G}_{2} / \mathrm{M}$ phase cells were 10.34 and $22.8 \%$ in the 5 and $10 \mu \mathrm{M}$ PEITC groups, respectively,

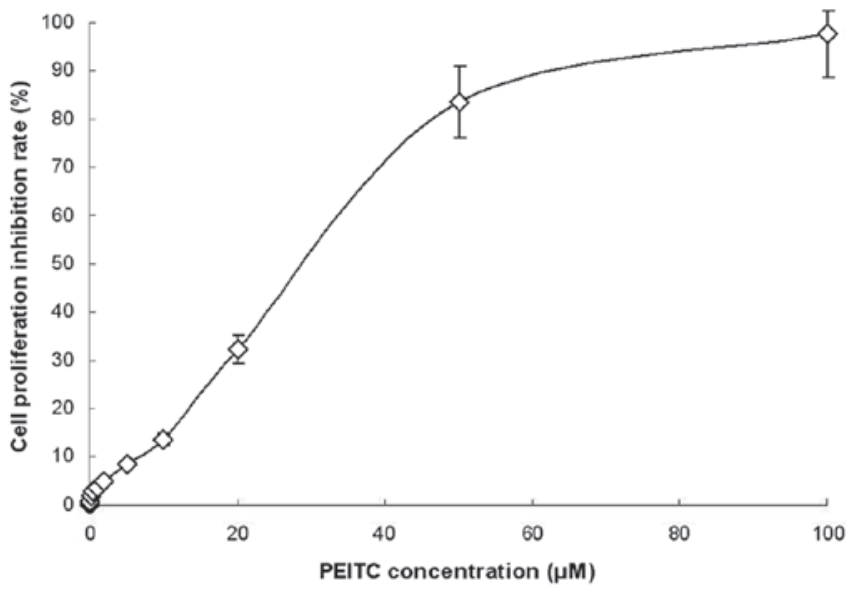

Figure 1. Effect of PEITC on the proliferative potential of HeLa cells, using the Vi-CELL cell viability analyzer. The data are presented as the means \pm standard deviation. ${ }^{*} \mathrm{P}<0.05, \mathrm{n}=5$. PEITC, phenethyl isothiocyanate.

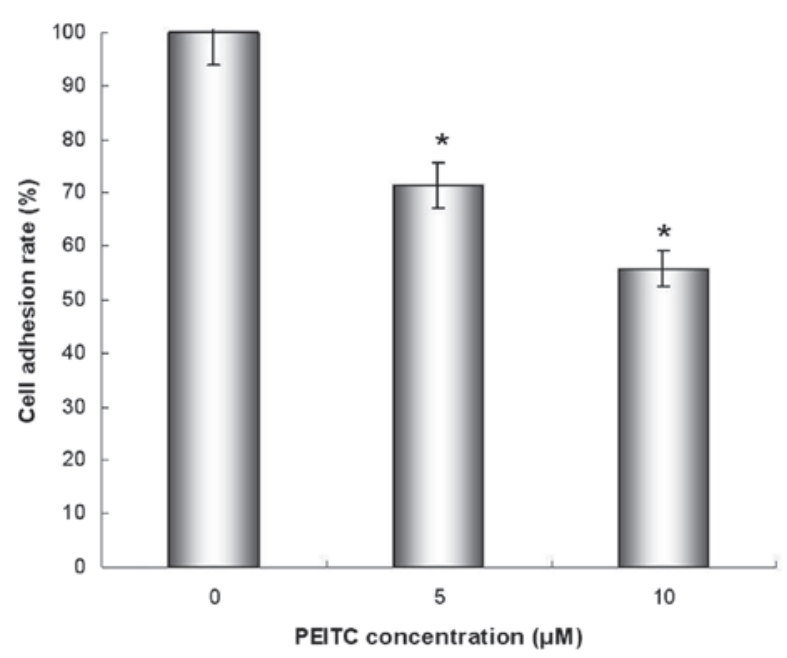

Figure 2. Effect of PEITC on the adhesive potential of HeLa cells, as determined by cell adhesion assay. The data are expressed as the means \pm standard deviation. ${ }^{*} \mathrm{P}<0.05, \mathrm{n}=5$. PEITC, phenethyl isothiocyanate.

and $3.65 \%$ in the control group $(\mathrm{P}<0.05)$. PEITC significantly induced cell cycle arrest at the $\mathrm{G}_{2} / \mathrm{M}$ phase.

PEITC reduces the production of TGF- $\beta, I L-6$ and $I L-8$ in HeLa cells. To observe the effects of PEITC on TGF- $\beta$, IL-6 and IL- 8 production, the expression levels of TGF- $\beta$, IL- 6 and IL-8 were examined by ELISA. The results indicated that the production of TGF- $\beta$, IL- 6 and IL- 8 was significantly reduced in HeLa cells incubated with 5 or $10 \mu \mathrm{M}$ PEITC, as compared with the control group (Fig. 5).

PEITC downregulates metastasis-related genes and proteins in HeLa cells. CDK1, MMP-2/9, CD44 and ICAM-1 all function in cervical cancer metastasis. The mRNA and protein expression levels of CDK1, MMP-2/9, CD44 and ICAM-1 were investigated. The results indicated that cells incubated with 5 or $10 \mu \mathrm{M}$ PEITC exhibited reduced mRNA and protein levels of CDK1, MMP-2/9, CD44 and ICAM-1, compared with the control group (Fig. 6). These results were consistent with the assays for adhesion, invasion and cell cycle. 

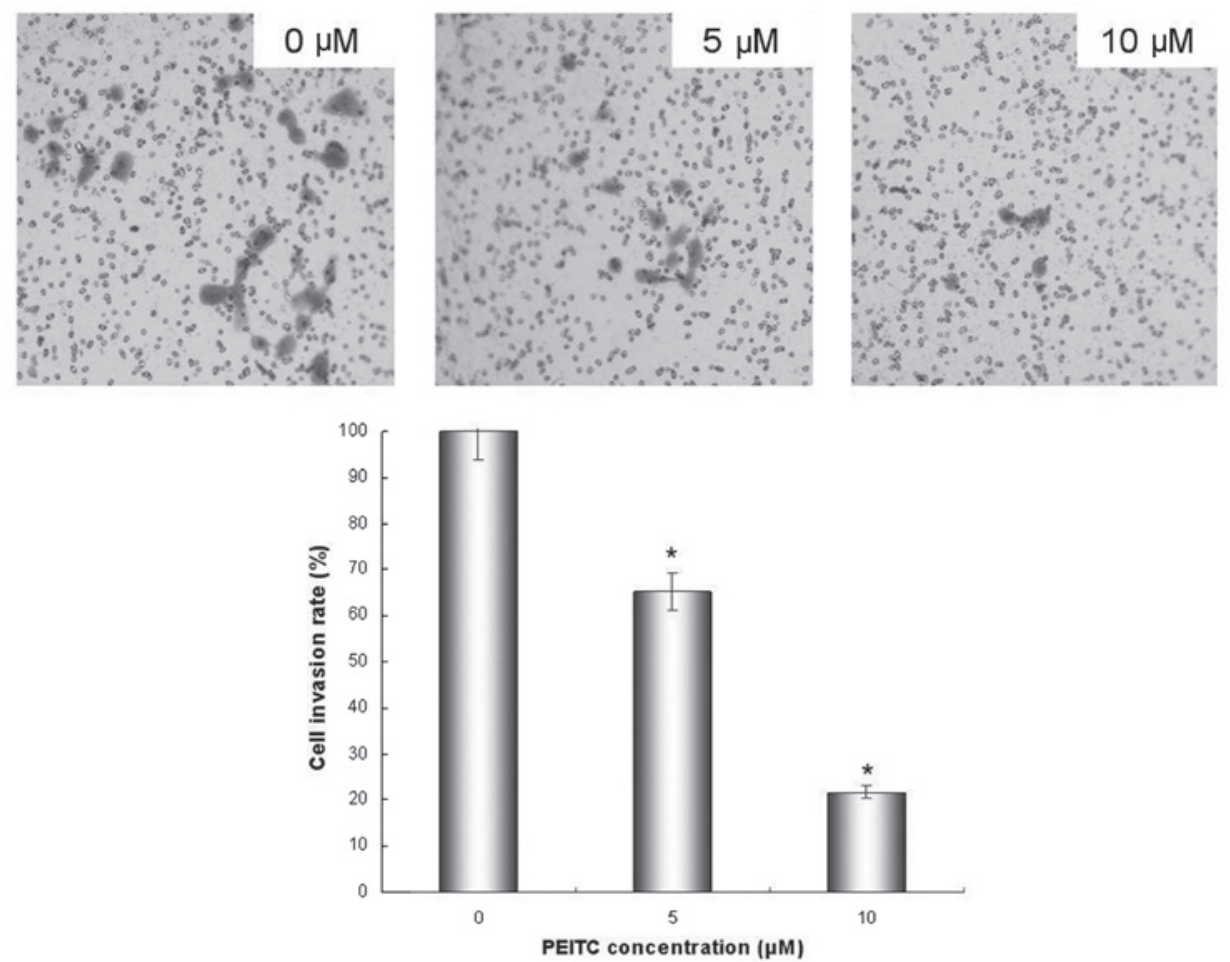

Figure 3. Effect of PEITC on the invasive potential of HeLa cells, as determined by Transwell assay (magnification, $\mathrm{x} 40$ ). The data are presented as the mean \pm standard deviation. ${ }^{*} \mathrm{P}<0.05, \mathrm{n}=3$. PEITC, phenethyl isothiocyanate.
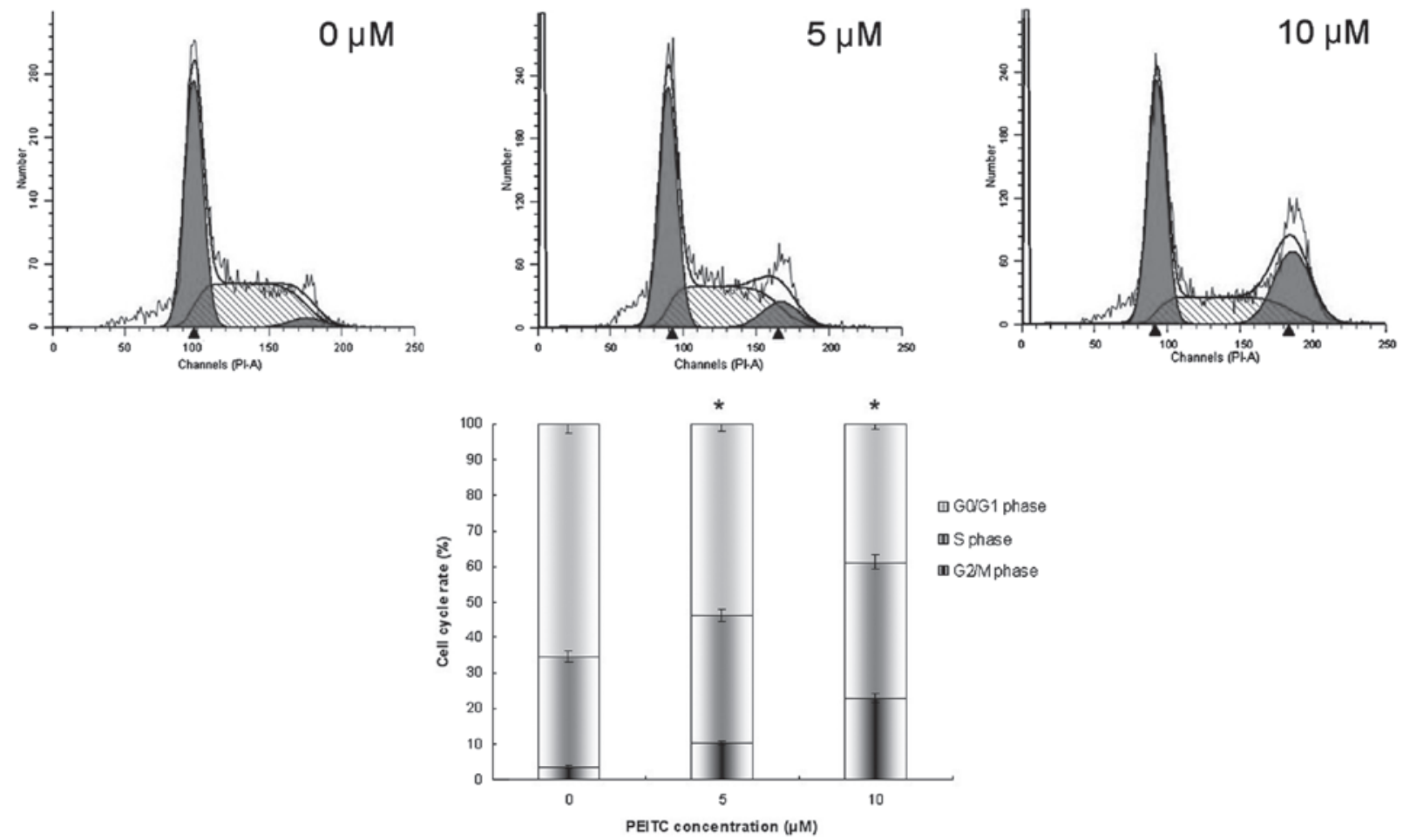

Figure 4. Effect of PEITC on the cell cycle of HeLa cells, as determined by flow cytometry. The data are presented as the means \pm standard deviation. "P<0.05, $\mathrm{n}=3$. PEITC, phenethyl isothiocyanate.

PEITC alters the metastasis-related signal transduction pathway of HeLa cells. Smad2 is an important cell signaling molecule. To evaluate whether Smad2 is target of PEITC in the inhibition of cervical carcinoma cell metastasis, Smad2 phosphorylation was detected by western blotting. PEITC reduced the phosphorylation of Smad2 in a dose-dependent manner in HeLa cells incubated with 5 or $10 \mu \mathrm{M}$ PEITC, as compared with the control group (Fig. 7). 


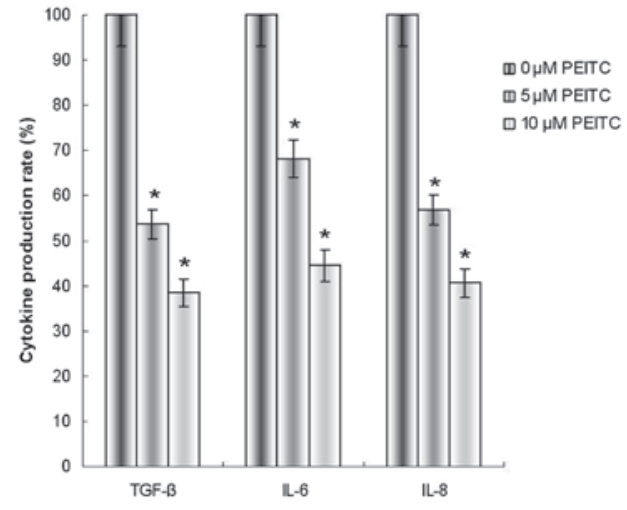

Figure 5. Effect of PEITC on the cytokine production of HeLa cells. TGF- $\beta$, IL- 6 and IL-8 production of HeLa cells was determined by ELISA. The data are presented as the means \pm standard deviation. "P $<0.05, n=5$. PEITC, phenethyl isothiocyanate; TGF, transforming growth factor; IL, interleukin.

A

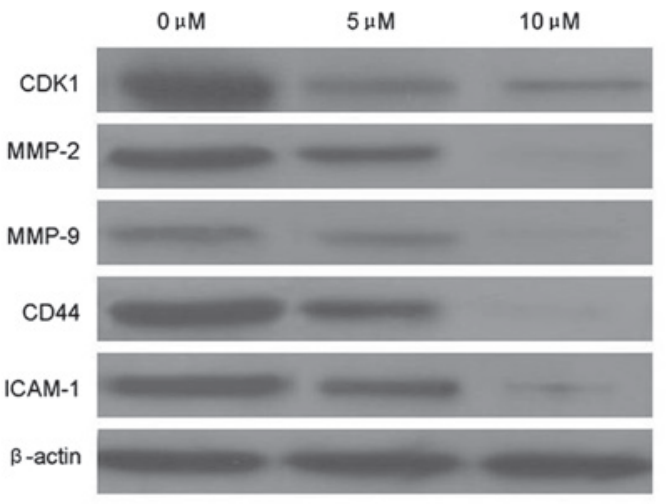

B

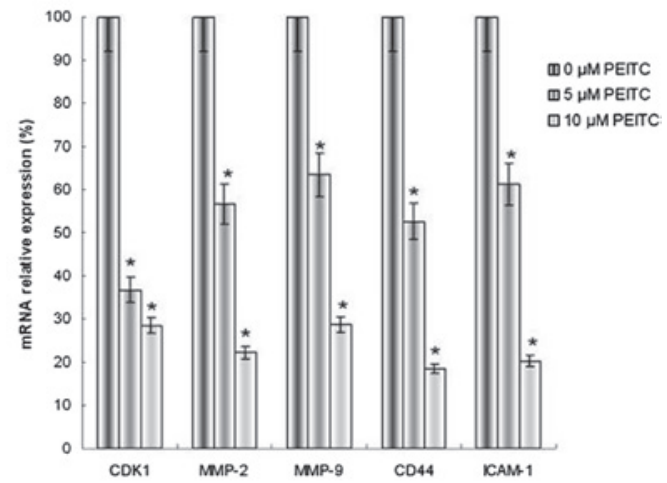

Figure 6. Effect of PEITC on the modulation of CDK1, MMP-2/9, CD44 and ICAM-1 metastasis-related genes in HeLa cells. (A) Protein expression, as determined by western blotting with $\beta$-actin as an internal control for loading, and (B) mRNA expression, determined by quantitative polymerase chain reaction, with GAPDH as an internal control for loading. The data are presented as the means \pm standard deviation. ${ }^{*} \mathrm{P}<0.05, \mathrm{n}=5$. PEITC, phenethy isothiocyanate; CDK, cyclin-dependent kinase; MMP, matrix metalloproteinase; ICAM, intercellular adhesion molecule.

$\mathrm{O} \mu \mathrm{M}$

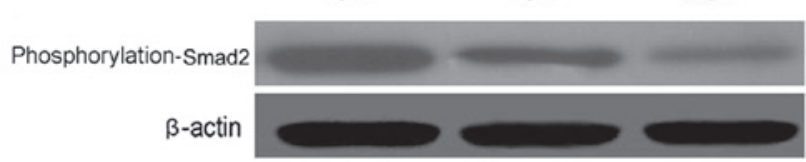

Figure 7. Effect of PEITC on the metastasis-related signal transduction pathway of HeLa cells. The phosphorylation of Smad2 was determined by western blotting. $\beta$-actin was used as an internal control for loading. PEITC, phenethyl isothiocyanate; $\operatorname{Smad} 2$, smad family member 2.

\section{Discussion}

PEITC, a constituent of cruciferous plants and a member of the isothiocyanate family, has been identified to have antitumor properties. The development of novel anti-metastatic drugs is currently one of the most important areas of tumor research. It has been reported recently that PEITC serves an import function in anti-metastatic processes in various tumor cells, including human hepatoma, human colon cancer and breast carcinoma cells (9-11). This effect is associated with the regulation of the expression of metastasis-related genes, cytokines and signal transduction molecules. However, little is known regarding the potential of PEITC in cervical cancer. In the present study, the effects of PEITC on cervical carcinoma cell metastasis potential was evaluated by adhesion and invasion assays following treatment with two concentrations of PEITC. The results suggested that PEITC effectively inhibited cervical carcinoma cell metastasis in vitro.

The effect of PEITC on metastasis-related gene expression was then evaluated. MMPs are members of a superfamily of zinc endopeptidases, and MMP-2 and MMP-9 are hypothesized to be crucial in the degradation of extracellular matrix due to their ability to cleave type IV collagen (12). CD44 and ICAM-1 are important adhesion molecules that induce heterogeneous cell adhesion and polarity alteration $(13,14)$. The data indicated that PEITC reduced MMP-2, MMP-9, CD44 and ICAM- 1 mRNA and protein expression levels. TGF- $\beta$, IL-6 and IL- 8 have been indicated to be important cytokines that serve a critical function in cancer invasion and metastasis (15). In the present study, reduced production of these cytokines was observed, indicating that PEITC has the ability to inhibit the metastasis of cervical carcinoma through the inhibition of TGF- $\beta$, IL- 6 and IL- 8 production.

A possible mechanism by which PEITC may protect against cancer is through cell cycle arrest. PEITC has been demonstrated to suppress growth and metastasis by this method in lung, prostate and ovarian cancer. In the present study, the results indicated that PEITC induced a dramatic accumulation of HeLa cells in the $\mathrm{G}_{2} / \mathrm{M}$ phase. It was previously reported that CDK1 regulates the cell cycle in mammalian cells, and is activated primarily in $\mathrm{G}_{2} / \mathrm{M}$ phase progression (16). Results of the present study also indicated that PEITC reduced the protein levels of CDK1, which suggests that PEITC induced cell cycle arrest by suppressing the expression of CDK1.

It is well-established that the Smad2 activation pathway is a major cell survival pathway involved in immunity, stress responses, inflammation and the inhibition of apoptosis and metastasis $(17,18)$. In the present study, the effect of PEITC on Smad2 phosphorylation was examined. It was demonstrated that PEITC inhibited Smad2 phosphorylation in a dose-dependent manner, suggesting that $\mathrm{p}$-Smad2 is a potential target of PEITC.

The present study provides support to the hypothesis that PEITC promotes strong inhibition of metastasis by blocking the TGF- $\beta /$ Smad2 pathway, which leads to the inhibition of expression of metastasis-related genes in HeLa cells. Understanding the mechanism of action of PEITC may provide valuable information for its possible application in anti-tumor therapy. A number of studies have evaluated the effects of PEITC in human subjects, and the present study may potentially facilitate the clinical development of isothiocyanates for cancer therapy. 


\section{Acknowledgements}

This study was supported by Tianjin Medical University Science Fund (2012KYM04).

\section{References}

1. Jemal A, Bray F, Center MM, Ferlay J, Ward E and Forman D: Global cancer statistics. CA Cancer J Clin 61: 69-90, 2011.

2. Wen Y, Pan XF, Zhao ZM, Chen F, et al: Knowledge of human papillomavirus (HPV) infection, cervical cancer, and HPV vaccine and its correlates among medical students in southwest China: a multi-center cross-sectional survey. Asian Pac J Cancer Prev 15: 5773-5779, 2014.

3. Ortega-Calderón YN and López-Marure R: Dehydroepiandrosterone inhibits proliferation and suppresses migration of human cervical cancer cell lines. Anticancer Res 34: 4039-4044, 2014.

4. Kidd EA, Siegel BA, Dehdashti F, Rader JS, et al: Lymph node staging by positron emission tomography in cervical cancer: relationship to prognosis. J Clin Oncol 28: 2108-2113, 2010.

5. Wolf MA and Claudio PP: Benzyl isothiocyanate inhibits HNSCC cell migration and invasion, and sensitizes HNSCC cells to cisplatin. Nutr Cancer 66: 285-294, 2014.

6. Wang Y, Wei S, Wang J, Fang Q and Chai Q: Phenethyl isothiocyanate inhibits growth of human chronic myeloid leukemia K562 cells via reactive oxygen species generation and caspases. Mol Med Rep 10: 543-549, 2014.

7. Zhu Y, Zhuang JX, Wang Q, Zhang HY and Yang P: Inhibitory effect of benzyl isothiocyanate on proliferation in vitro of human glioma cells. Asian Pac J Cancer Prev 14: 2607-2610, 2013.

8. Yan H, Zhu Y, Liu B, Wu H, Li Y, Wu X, Zhou Q and Xu K. Mitogen-activated protein kinase mediates the apoptosis of highly metastatic human non-small cell lung cancer cells induced by isothiocyanates. Br J Nutr 106: 1779-1791, 2011.
9. Hunakova L, Sedlakova O, Cholujova D, Gronesova P, Duraj J and Sedlak J: Modulation of markers associated with aggressive phenotype in MDA-MB-231 breast carcinoma cells by sulforaphane. Neoplasma 56: 548-556, 2009.

10. Hwang ES and Lee HJ: Benzyl isothiocyanate inhibits metalloproteinase-2/-9 expression by suppressing the mitogen-activated protein kinase in SK-Hep1 human hepatoma cells. Food Chem Toxicol 46: 2358-2364, 2008.

11. Lai KC, Huang AC, Hsu SC, et al: Benzyl isothiocyanate (BITC) inhibits migration and invasion of human colon cancer HT29 cells by inhibiting matrix metalloproteinase-2/-9 and urokinase plasminogen (uPA) through PKC and MAPK signaling pathway. J Agric Food Chem 58: 2935-2942, 2010.

12. Groblewska M, Siewko M, Mroczko B and Szmitkowski M: The role of matrix metalloproteinases (MMPs) and their inhibitors (TIMPs) in the development of esophageal cancer. Folia Histochem Cytobiol 50: 12-19, 2012.

13. Kung CI, Chen CY, Yang CC, et al: Enhanced membrane-type 1 matrix metalloproteinase expression by hyaluronan oligosaccharides in breast cancer cells facilitates CD44 cleavage and tumor cell migration. Oncol Rep 28: 1808-1814, 2012.

14. Kwiatkowska A and Symons M: Signaling determinants of glioma cell invasion. Adv Exp Med Biol 986: 121-141, 2013.

15. Yigit R, Massuger LF, Zusterzeel PL, et al: Cytokine profiles in cyst fluids from ovarian tumors reflect immunosuppressive state of the tumor. Int J Gynecol Cancer 21, 1241-1247, 2011.

16. Lamberto I, Plano D, Moreno E, et al: Bisacylimidoselenocarbamates cause $\mathrm{G} 2 / \mathrm{M}$ arrest associated with the modulation of CDK1 and Chk2 in human breast cancer MCF-7 cells. Curr Med Chem 20: 1609-1619, 2013.

17. Lu D, Han C and Wu T: 15-hydroxyprostaglandin dehydrogenase-derived 15-keto-prostaglandin E2 inhibits cholangiocarcinoma cell growth through interaction with peroxisome proliferator-activated receptor- $\gamma$, SMAD $2 / 3$, and TAP63 proteins. J Biol Chem 288: 19484-19502, 2013.

18. Huang C, Shen S, Ma Q, et al: Blockade of KCa3.1 ameliorates renal fibrosis through the TGF- $\beta 1 /$ Smad pathway in diabetic mice. Diabetes 62: 2923-2934, 2013. 